\title{
Identification
}

\section{des implants dentaires \\ en odontologie médico-légale}

\section{RÉSUMÉ}

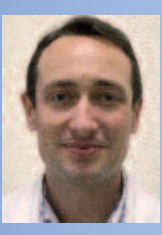

François-Georges WEMEAU

Praticien hospitalier,

CH Roubaix. Stomatologie,

CHRU Lille. Psychiatrie, médecine légale

et médecine en milieu pénitentiaire

Centre Hospitalier de Roubaix

Hôpital Victor Provo,

11 boulevard Lacordaire

59100 Roubaix.

Centre Hospitalier Régional Universitaire de Lille

2, avenue Oscar Lambret,

59037 Lille Cedex.

Hugues THOMAS

Expert en identification odontologique près la cour d'appel de Nancy.

Directeur de mémoire.

Aline SUYBENG

Chirurgien-dentiste.

\section{Aimé CONIGLIARO}

Chef par suppléance du département Anthropologie-Thanatologie-OdontologieIRCGN.

L'implantologie dentaire est une discipline qui connaît depuis les années 1990 une expansion conséquente. Néanmoins, il n’y a pas de marquage pour ces implants, à la différence d'autres comme les implants orthopédiques, et peu de documentation existe sur le thème de leur identification.

Notre étude s'est intéressée à l'élaboration d'une méthodologie devant la découverte d'un implant, seul ou dans un corps, avec les radiographies retro-alvéolaires et panoramiques, avec ou sans dépose des maxillaires, et éventuellement « implant en main ».

De par leur type et leur présence, nous avons alors découvert quelles informations étaient associées : état de santé du porteur de l'implant, statut social, âge de l'implant... Les moyens d'analyse de ces implants dentaires ont ensuite été présentés à travers l'analyse radiologique, et par les bases de données par les sites « what implant is that » et le système IRS (Implant Recognition System). Enfin, nous avons élaboré une étude de ces implants avec la réalisation de fiches identificatrices et un logiciel qui permet de sélectionner de 1 à 3 paramètres pour retrouver des candidats potentiels.

Cette étude sur l'identification des implants dentaires met en évidence la difficulté de son analyse et semble confirmer la nécessité d'une formation en implantologie et en odontologie légale. Il faut éviter les erreurs et les approximations qui orienteraient mal les logiciels. L'explosion des types d'implants existants (et notamment le problème de copies) rend cette recherche ardue. Ainsi il est obligatoire de mettre à jour régulièrement et de compléter les bases de données existantes, ceci étant un travail complexe et fastidieux.

- implantologie

- identification odontologie légale

- médecine légale

- logiciel de reconnaissance
AOS 2012;260:341-351

DOI: $10.1051 / a 0 s / 2012405$

(C) EDP Sciences 


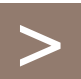

L'odontologie médico-légale se définit comme l'interface entre l'odontologie et la justice. Elle s'appuie sur une méthodologie fiable et objective qui justifie pleinement son existence et son extension à coté des empreintes digitales ou de l'analyse ADN [13, 14].

L'identification des implants dentaires fait partie des différentes missions de l'odontologie médico-légale.

Avec l'essor de l'implantologie, nous avons une nouvelle source d'information qu'il faut savoir exploiter.
Les implants ont une haute résistance mécanique, thermique et sont très stables dans le temps $[2,9,20]$.

En revanche, il n'existe pas de marquage pour ces implants dentaires, à la différence d'autres implants comme les implants orthopédiques, et peu de documentation existe sur ce thème d'identification.

C'est le but de ce travail : potentialiser les informations véhiculées par les implants pour permettre une identification odontologique.

\section{Quelle méthodologie pour les implants dentaires?}

Une panoramique dentaire ou orthopantomogramme est effectuée afin de rédiger entre autres l'odontogramme. Des rétroalvéloaires peuvent également être réalisées.
Plus fines, elles peuvent donner une image de I'implant dans le site osseux qui sera une aide à la reconnaissance de l'implant dentaire (fig. 1).

\section{Quelles informations apportent la présence d'implant dentaire?}

De par leur seule présence, les implants peuvent donner de nombreux renseignements, comme l'état de santé de la personne. En effet, il existe des indications, mais aussi des contreindications, relatives ou absolues, quant à une pose implantaire (risque oslérien, infection focale...), limitée néanmoins à la date de celle$c i$, une pathologie pouvant toujours se développer ultérieurement $[1,15]$.

Les implants dentaires ne bénéficient pas en France de prise en charge par la sécurité sociale et font partie des actes non remboursés. C'est une thérapeutique qui reste onéreuse, donc même si cette technique se "démocratise ", elle reste assimilée à une population sans difficulté financière $[5,6]$.

La conception de l'implant peut également nous donner des indications quant à sa date de réalisation, voire son époque et sa répartition géographique $[4,7,8]$. Une sorte de " phénomène de mode " existerait aidant encore à estimer la date de pose et de fabrication de ces implants. Le plus important est qu'il y a automatiquement un dossier médical comportant des 


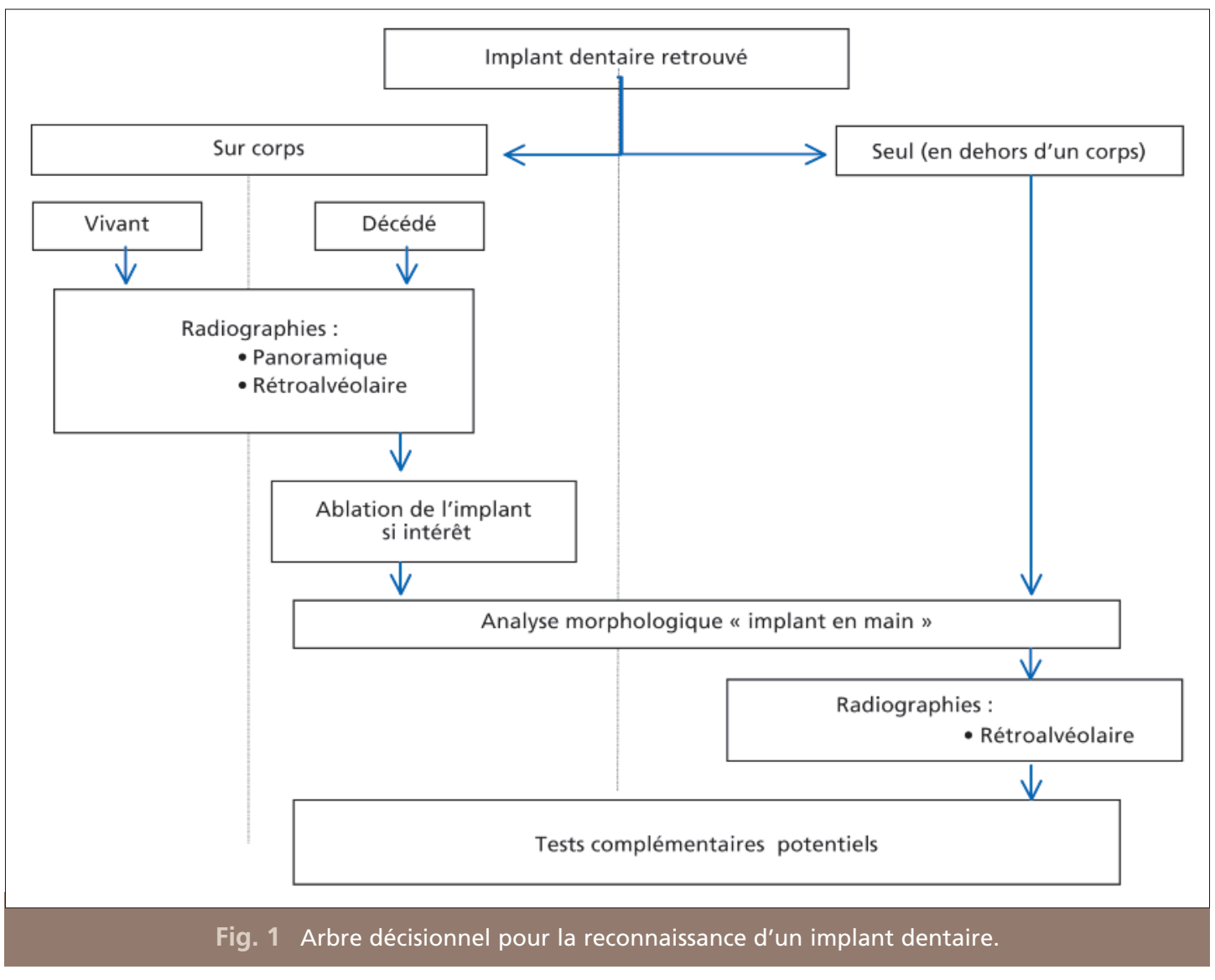

radiographies permettant un travail de comparaison. Identifier l'implant peut permettre d'orienter les recherches et de retrouver le dossier ante-mortem.

On peut aussi caractériser un implant par sa morphologie et ainsi le reconnaître globale- ment en regardant ses trois parties anatomiques : le col, le corps et l'apex.

On peut ainsi en dégager cinq types : cylindrique, transgingival, à col évasé, conique et cylindro-conique. Les implants peuvent également être filetés ou non filetés $[7,8]$.

\section{Analyse radiologique et bases de données : moyen d'exploitation}

En 1992, Sewerin a tenté par l'étude des radiographies d'implants dentaires selon différents axes de rotation de constituer des tableaux [19].
Ceux-ci devaient pouvoir aider à identifier l'influence des éléments variables: le filetage, les coupes, les trous, les perforations. Il a démontré 
l'importance d'établir des bases de données si I'on veut envisager cette identification implantaire.

Les travaux de Sahiwal en 2002 ont permis de mettre en place une classification morphologique [16-18]. Chaque critère déterminé permettrait de retrouver, par élimination ou par confirmation successive, les candidats potentiels pour la reconnaissance de l'implant.

Suite aux recherches de Sahiwal, une base de données s'est constituée et est apparue sous la forme d'un site internet : " what implant is that ? " (www.whatimplantisthat.com) (fig. 2). Il s'agit d'un site gratuit et en ligne qui collecte les informations entre autres sous forme de radiographies afin de réaliser une base de données. Le programme permet alors soit de retrouver un implant par rapport à des caractéristiques morphologiques, soit de voir quels seraient les implants dentaires validant des critères recherchés.

Le site présente alors des radiographies que l'examinateur peut enfin comparer avec ses propres radiographies. Tout ceci a été mis en place pour faciliter l'identification implantaire.

Le demandeur doit renseigner trois critères liés à l'anatomie de l'implant:

- la partie coronaire (« coronal ») ;

- la partie intermédiaire (" mid-body») ;

- l'apex (« apical »).

Tous ces renseignements tendent à être le plus précis possible mais, à l'inverse, ont l'inconvénient de fausser le moteur de recherche si les champs sont mal remplis. Ainsi, il est conseillé de ne pas mettre trop de renseignements dans un premier temps.

Cette étude et recherche peut sembler également difficile pour quelqu'un non habitué aux implants dentaires ou qui n'aurait pas réussi à identifier seul l'implant en question sur le site. Autre cas de figure, le demandeur n'aurait pas le temps de réaliser cette recherche : dans ce cas, un autre volet existe sur ce site afin de demander à des intervenants de retrouver le type d'implant que I'on cherche. Cette démarche est alors payante (75 dollars par recherche en 2011). Dans ce cas, il faut envoyer
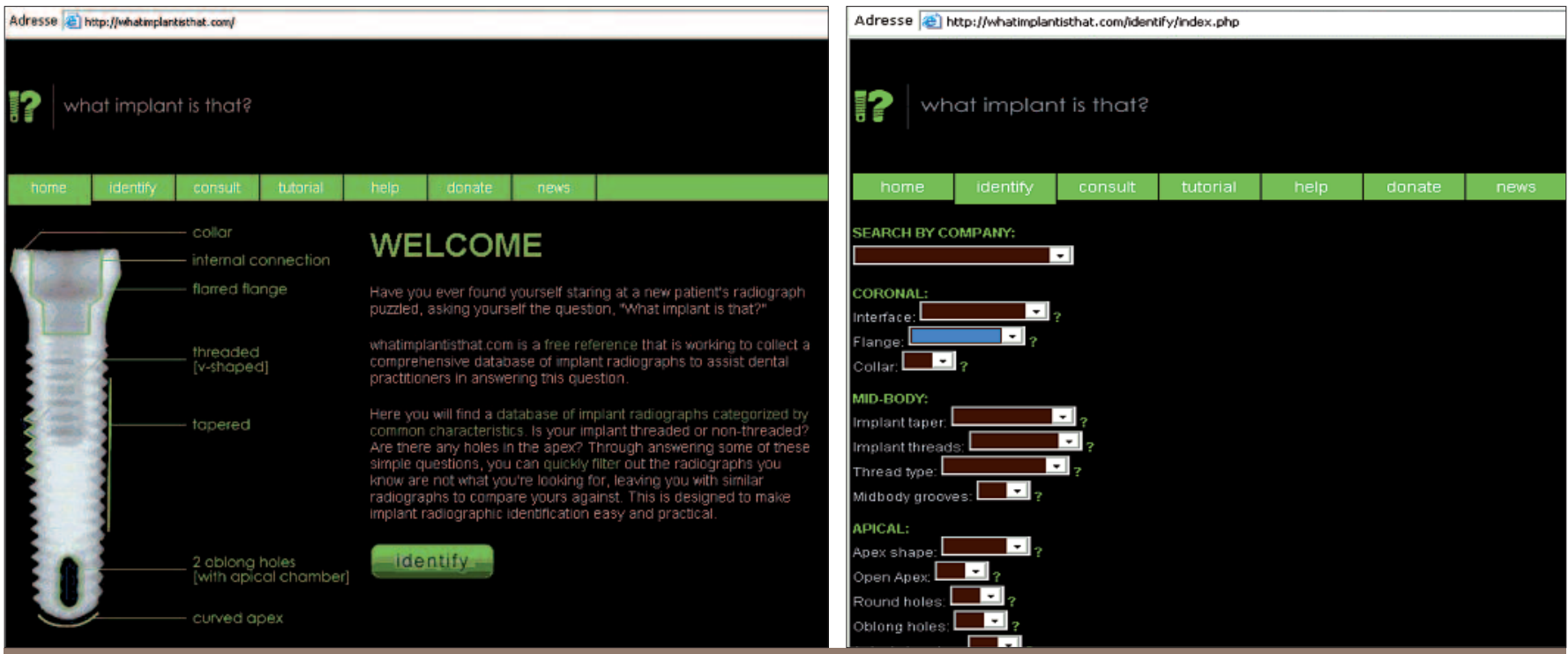

Fig. 2 a et b Page d'accueil et méthode de recherche sur www.whatimplantisthat.com 
la photographie ou l'image de la radiographie (rétroalvéolaire de préférence). Le cliché doit être le plus perpendiculaire possible à l'axe long de l'implant afin de voir des aspects importants comme le type de fil, les rainures, etc.

Un autre site existe aussi, avec le système IRS (Implant Recognition System) : " which implant » (www.whichimplant.com) (fig. 3).

En 2006, Michelinakis et al. ont développé un logiciel informatique permettent d'aider l'identification implantaire des implants in vivo en utilisant une série de questions [11]. Le site est payant et il faut s'inscrire au préalable en remplissant la fiche de renseignement. Le coût pour un abonnement annuel est de 30 livres sterling soit environ 34 euros.

Ce logiciel d'identification d'implants dentaires fonctionne sur le principe d'un ensemble de données stockées dans une base autonome. Plusieurs questions sont utilisées pour détecter les candidats possibles. L'IRS pose ainsi neuf questions : deux d'ordre général puis sept s'intéressant à des critères morphologiques spécifiques. Les deux premières cases $d$ 'ordre général permettent d'indiquer le nom du laboratoire de l'implant, puis le nom de l'implant. Cette recherche rapide donne la possibilité de retrouver rapidement et simplement les caractéristiques d'un implant que I'on connaît ou que l'on aimerait voir.

Les sept autres questions se portent sur la morphologie de l'implant :

- le type d'implant, par exemple cylindrique, avec chambre apicale ;

- la description (du type de connexion), par exemple : octogonale interne ;

- fileté ou non ;

- le type de surface ;

- le type de col ;

- le diamètre ;

- la longueur.
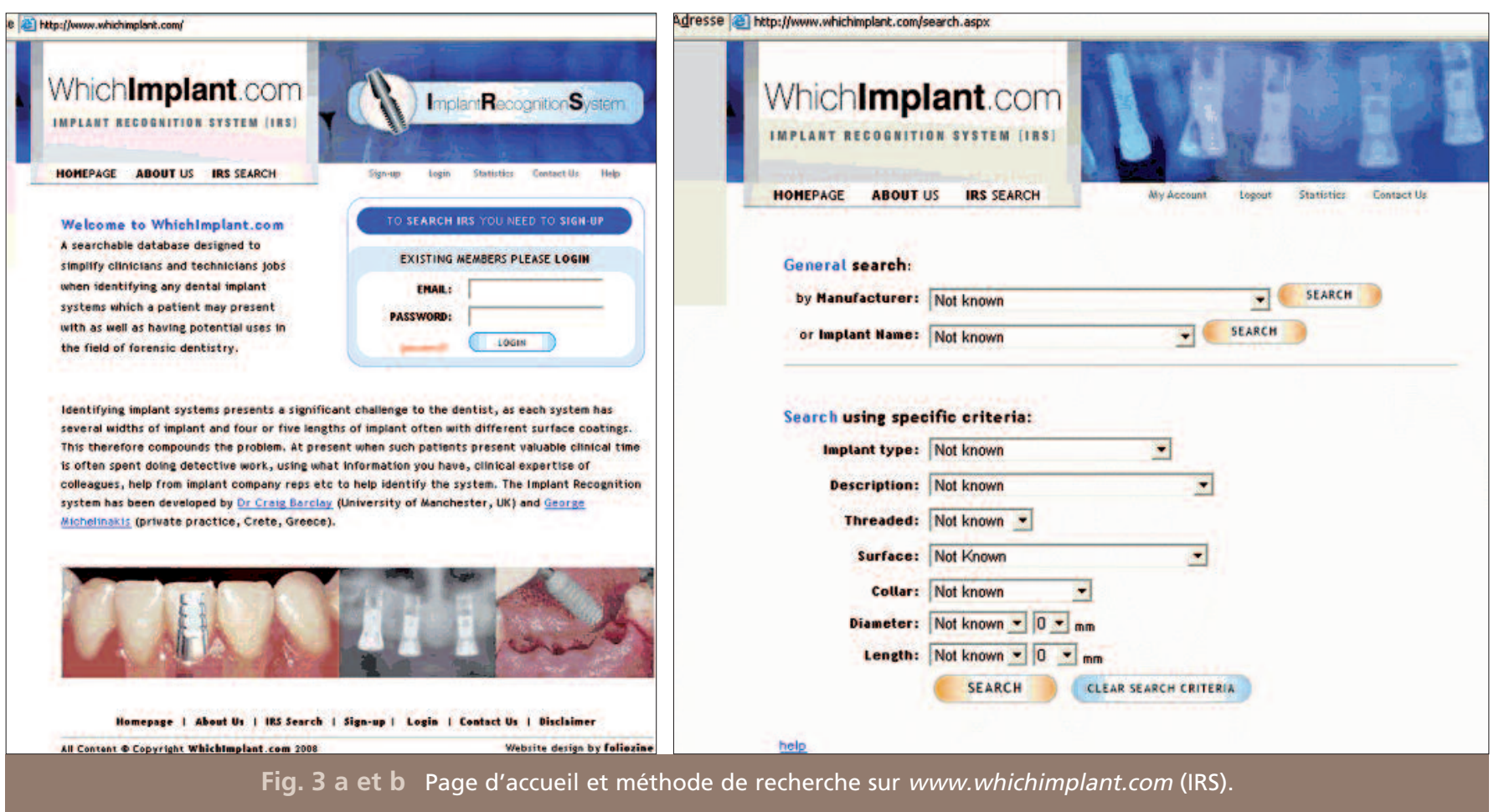

Fig. 3 a et b Page d'accueil et méthode de recherche sur www.whichimplant.com (IRS). 
Comme précédemment, en indiquant trop de critères, on risque de fausser la recherche. II vaut mieux donner moins de critères si l'on n'est pas certain de ceux-ci.

Après les avoir déterminés, on lance la recherche et les candidats apparaissent sous forme de tableaux. On a alors le nom du laboratoire et le nom de l'implant. Une troisième case écrite en bleu permet d'avoir d'autre détail sur l'implant et de basculer sur sa fiche.

Chaque fiche présente les coordonnées du fabricant ainsi que le site Internet, la description de l'implant et les différentes longueurs et diamètres existants.

Pour que cette base de données puisse être cliniquement utile dans le long terme, la banque

\section{Travail personnel ${ }_{[21]}$}

Pour notre étude, nous nous sommes intéressés aux implants dentaires " implant en main » afin d'essayer de constituer un début de base de données plus morphologique en parallèle des informations radiologiques existantes.

Les informations recueillies afin de créer ce travail ont été récupérées selon successivement trois modes :

- rencontres et/ou appels téléphoniques avec les visiteurs médicaux des laboratoires concernés :

- courriels envoyés aux laboratoires et aux praticiens pratiquant l'implantologie ;

- sites Internet.

La majorité des renseignements ont été récupérés sur les sites Internet, ceux-ci pour la plupart étant d'ailleurs assez complets. Il est à déplorer le peu de réponses des laboratoires (nous orientant souvent vers leur site puisque notre étude n'envisageait pas d'achat d'im- de données des systèmes d'implant est mise à jour annuellement.

Déjà en 2008, Nuzzolese avait établi I'avantage des bases de données dans la recherche et I'identification des implants dentaires en médecine légale [12].

L'intervention de I'IRS a été estimée par la suite lors d'une étude de Berketa en 2010 et son intérêt a bien été confirmé, même si la formation de l'utilisateur reste primordiale [3].

La recherche peut ainsi se faire en suivant une méthode globale ou générale afin de trouver le type d'implant (type de compagnie ou d'industrie, nom de l'implant...), ou en cherchant critère par critère les caractéristiques de l'implant (forme générale, apex, fileté ou non...).

plant), et encore moins de retours de nos confrères implantologistes.

Les laboratoires et les implants concernés par cette étude correspondent aux implants les plus vendus en France selon une étude de Grully et Torres du service d'odontologie du CHRU de Montpellier [10]. Le marché français (et dans le monde) serait dominé par le " big six " à savoir (en \% de part du marché en France en 2008) : Nobel Biocare (31,3\%), Straumann $(13,4 \%)$, Zimmer Dental (12,2 \%), Dentsply $(11,6 \%)$, Biomet $3 \mathrm{i}(10,3 \%)$ et Astra Tech $(5,4 \%)$. Nous avons donc débuté cette étude par ces six industriels, pour après nous intéresser aux autres laboratoires. Au final, 90 implants venant de 20 laboratoires ont été étudiés.

Ici, nous sommes intéressés aux trois parties principales d'un implant :

- le corps,

- l'apex, 
- le col (et sa connexion).

Les résultats ont pu être regroupés sur un fichier Excel. Grace à ce fichier faisant office de base de données, on peut soit rechercher critère par critère l'implant que l'on désire (7 cri- tères + l'image et la radio) ou alors on peut choisir de 1 à 3 paramètres pour trouver le ou les candidats. Pour de nombreux implants, des liens existent pour retrouver l'image et la radiographie correspondantes (fig. 4).

\begin{tabular}{|c|c|c|c|c|c|c|c|c|c|}
\hline 질 & Eichier Edition Affichage & Format Qutils & Fenêtre ? & & & & & \\
\hline \multicolumn{8}{|c|}{ 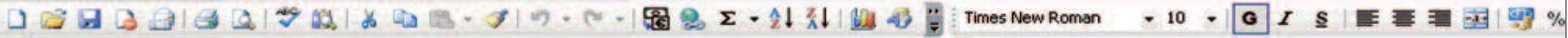 } & & \\
\hline \multicolumn{10}{|c|}{ 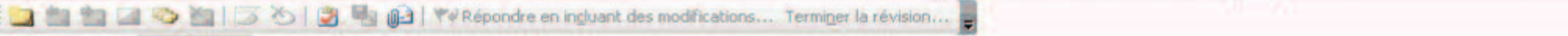 } \\
\hline & $\mathrm{H} 53 \rightarrow f_{x}$ & & & & & & & & \\
\hline & A & $B$ & C & D & $E$ & $\mathrm{~F}$ & G & $\mathrm{H}$ & 1 \\
\hline 1 & Marque de l'implant & Reference & Corps & Apex & Col & Type de connexio - & Fileté - & Images & Radios \\
\hline 48 & Neoss & ProActive & $\begin{array}{l}\text { Cylindrique à apex } \\
\text { rétréci }\end{array}$ & Plat & Droit & $\begin{array}{l}\text { Interne à } 6 \text { rainures } \\
\text { carrées }\end{array}$ & oui & neoss & neoss \\
\hline 49 & Nobel Biocare & Nobel active & Conique & Arrondi & Rétréci et fileté & Interne conique & oui & $\frac{\text { nobelbiocar }}{\text { nobelactive }}$ & $\frac{\text { nobelbiocar }}{\text { nobelactive }}$ \\
\hline 50 & Nobel Biocare & NobelReplace & Conique & Arrondi & Droit et lisse & Interne tri-lobée & oui & $\frac{\frac{\text { nobelbiocar }}{\mathrm{e}}}{\frac{\mathrm{e}}{\mathrm{e}}}$ & $\frac{\frac{\text { nobelbiocar }}{\text { nobelreplac }}}{\frac{\mathrm{e}}{\mathrm{e}}}$ \\
\hline 51 & Nobel Biocare & Brånemark System & Conique & Arrondi & Droit & Externe hexagonale & oui & $\begin{array}{l}\text { nobelbiocar } \\
\text { e- } \\
\text { branemark }\end{array}$ & \begin{tabular}{|c|} 
nobelbiocar \\
branemark \\
\end{tabular} \\
\hline 52 & Nobel Biocare & Nobelspeedy Replace & Conique & Plat & & Interne tri-lobée & oui & $\begin{array}{c}\frac{\text { nobelbiocar }}{\text { e }} \\
\text { nobelspeed } \\
\text { y }\end{array}$ & $\begin{array}{l}\frac{\text { nobelbiocar }}{\text { nobelspeed }} \\
\text { nober }\end{array}$ \\
\hline 53 & Nobel Biocare & Nobelspeedy Groovy & Conique & Plat & & Externe hexagonale & oui & & \\
\hline 54 & Osseolink & Osseolink & Cylindro-conique & Plat & Droit & Interne & oui & osseolink & osseolink \\
\hline 55 & Straumann & Tapered Effect & Conico-cylindrique & Arrondi & Evasé et lisse & Interne octogonale & oui & $\begin{array}{l}\text { straumann } \\
\text { tapered } \\
\text { effect }\end{array}$ & \begin{tabular}{|c|}
$\frac{\text { straumann }}{\text { effect }}$ \\
\end{tabular} \\
\hline 56 & Straumann & Standard & Cylindrique & Arrondi & Evasé et lisse & Interne octogonale & oui & $\begin{array}{l}\text { straumann } \\
\text { standard }\end{array}$ & $\begin{array}{l}\frac{\text { straumann }}{\text { standard }} \\
\end{array}$ \\
\hline 57 & Straumann & Standard Plus Narrow Neck & Cylindrique & Arrondi & Evasé et lisse & Externe octogonale & oui & $\begin{array}{l}\frac{\text { straumann }}{\text { narrow }} \\
\text { neck }\end{array}$ & $\frac{\text { straumann }}{\frac{\text { narrowy }}{\text { neck }}}$ \\
\hline 58 & Straumann & $\begin{array}{l}\text { Standard Plus Regular Neck } \\
\text { et Wide Neck }\end{array}$ & Cylindrique & Arrondi & Evasé et lisse & Interne octogonale & oui & $\frac{\frac{\text { straumann }}{\text { regulat }}}{\text { neck }}$ & $\frac{\frac{\text { straumann }}{\text { regular }}}{\text { neck }}$ \\
\hline
\end{tabular}
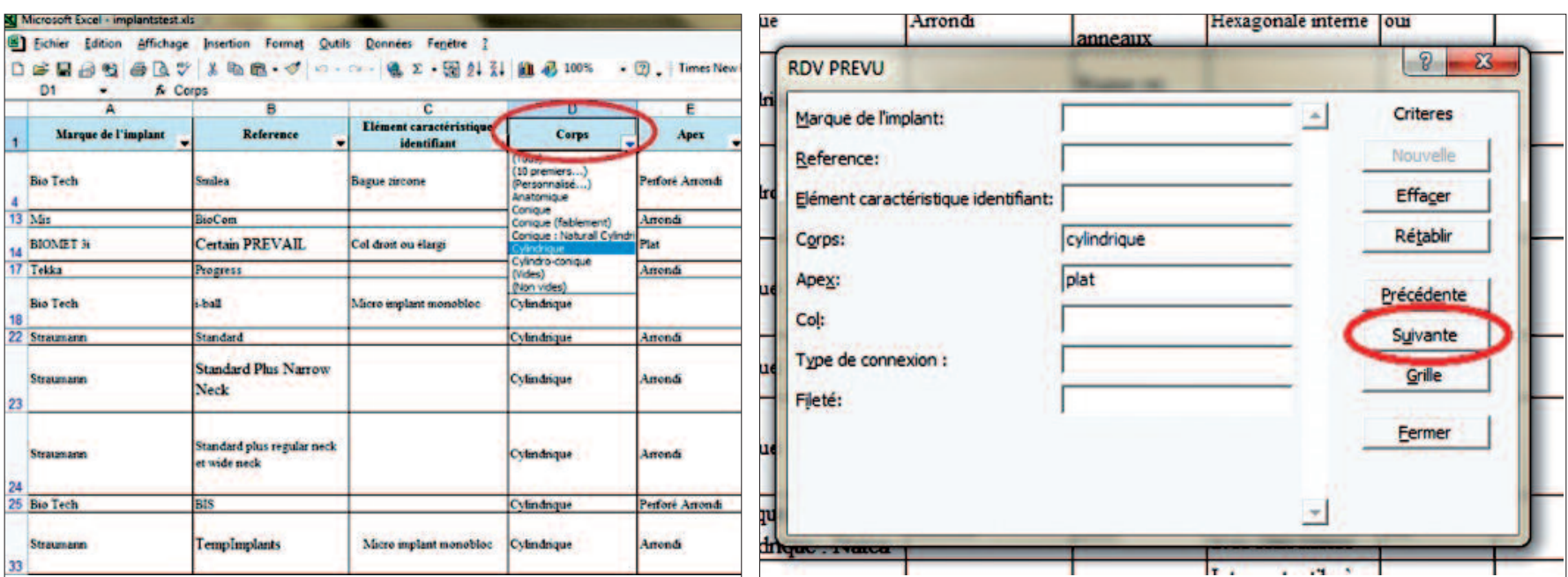

Fig. 4 a à c Présentation et méthode de recherche du logiciel Excel : deux possibilités de sélections des paramètres : soit en sélectionnant successivement les paramètres dans les différentes colonnes, soit en sélectionnant de 1 à 3 paramètres en passant par « Données », " Formulaire », " Critères ». 
Pour ce travail, une base de données a été créée, dans laquelle chaque implant est détaillé dans une fiche. Cette base de données riche de 120 implants est évolutive et peut être consultée sur demande (fig. 5). Ces fiches constituent un document facilement compulsable de type annuaire, mais qui peut être également visualisé sur le $\mathrm{CD}$. Ce travail vise à être une aide à l'identification implantaire.

C'est une étape préliminaire permettant une analyse " implant en main ». Elle viserait à confirmer l'identité d'un candidat avec ses fiches après une première recherche radiologique, ou alors présenter les premiers implants possibles si on a l'implant en main avant toute radiographie.

Ce fichier Excel n'a pas pour but de remplacer les autres bases de données déjà pertinentes existantes comme le sytème IRS ou " whatimplantisthat ", mais serait plutôt une aide afin de dégrossir en premier lieu la recherche envisagée.

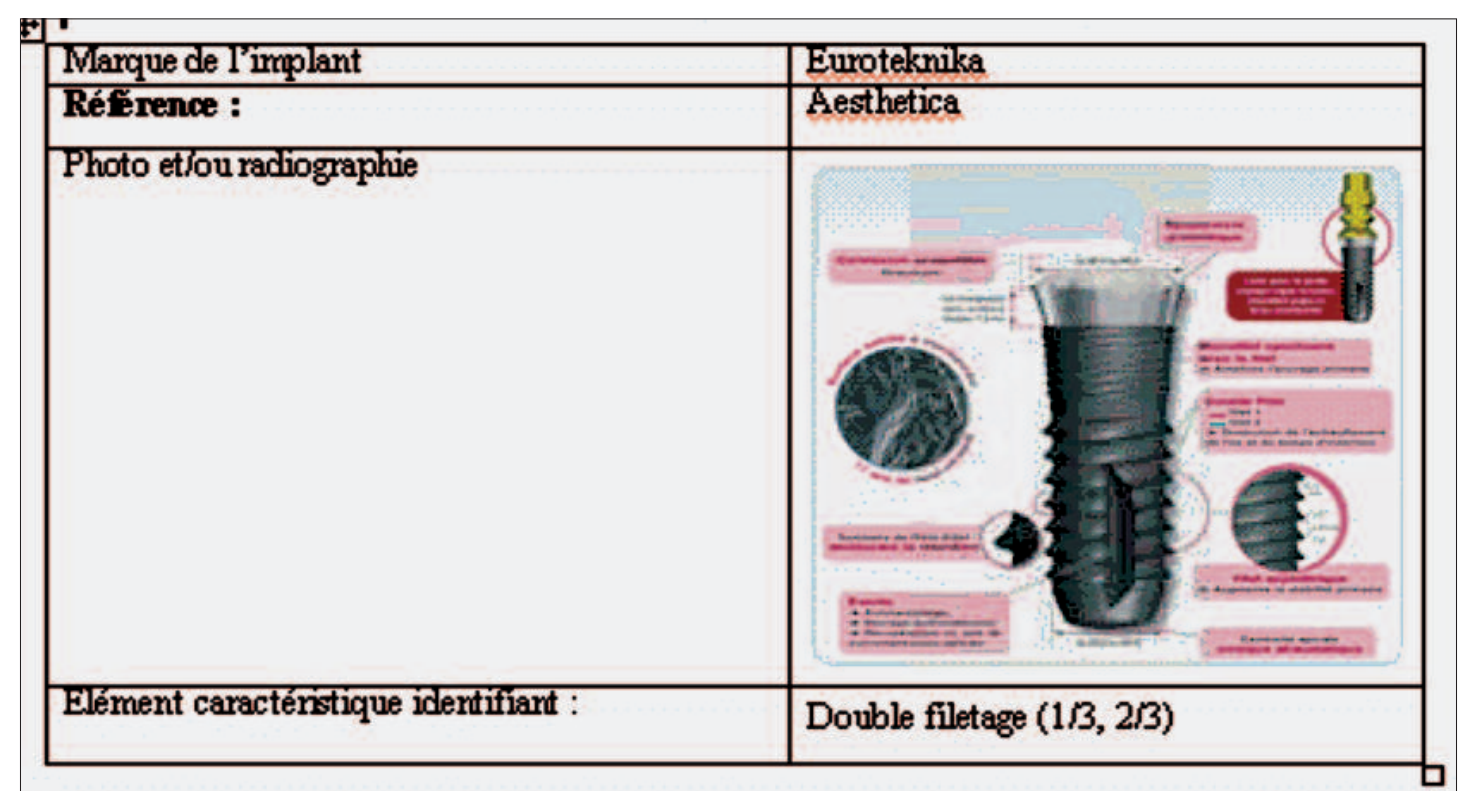

\begin{tabular}{|c|c|c|c|}
\hline Corps & Cocher le type & Apex & $\begin{array}{l}\text { Cocher le } \\
\text { type }\end{array}$ \\
\hline $\begin{array}{l}\text { Type d'implant: } \\
\text { Comique } \\
\text { Cylindrique } \\
\text { Cylindro- } \\
\text { comique } \\
\text { Autre type }\end{array}$ & Conique & $\begin{array}{l}\text { Type d'spex: } \\
\text { Perforé } \\
\text { Plein } \\
\text { Pli } \\
\text { Auroudi } \\
\text { Avrec rentlemert } \\
\text { Avtre type }\end{array}$ & Anondi \\
\hline $\begin{array}{l}\text { Fileté } \\
\text { Nonfileté } \\
\text { Autre }\end{array}$ & Fileté (couble filetage $1 / 3,2 / 3$ ) & & \\
\hline Cal & Lisse, évasé & & \\
\hline $\begin{array}{l}\text { Type de camexian: } \\
\text { interne } \\
\text { Externe } \\
\text { Hexagonale } \\
\text { Autre type }\end{array}$ & hexagonale inteme avec cône Morse & & \\
\hline
\end{tabular}

Fig. 5 Exemple de fiche réalisée. (Euroteknica. Aestheitica http://www.euroteknika.com/FRA). 


\section{Conclusion}

Cette étude sur l'identification des implants dentaires met évidence la difficulté de son analyse et semble confirmer la nécessité d'une formation en implantologie et en odontologie légale.

Même si les praticiens implantologistes ont une obligation de renseignements et doivent à la fois procurer une carte d'identité implantaire aux patients, tout en gardant cela dans leurs dossiers médicaux, cette recherche est souvent ardue du fait de l'absence ou " perte " de ces informations. L'examinateur est l'acteur principal et indispensable, des outils existent mais il est important de savoir comment les utiliser.ll faut éviter les erreurs et les approximations qui orienteraient mal les logiciels.
L'explosion des types d'implants - et notamment le problème de copies - rend cette recherche ardue. Pour être efficace, il est nécessaire de mettre à jour régulièrement et de compléter les bases de données existantes, travail complexe et fastidieux.

Initialement, ce travail avait été envisagé dans une optique d'identification médico-légale. Mais très vite nous nous sommes aperçus que les perspectives d'utilisation étaient bien plus larges. C'est un outil qui s'adresse en effet à tous les praticiens confrontés au problème de pourvoir renouveler des prothèses dentaires pour des nouveaux patients présentant des implants posés depuis de nombreuses années mais encore bien ostéo-intégrés.

\section{Bibliographie}

1. Assemat-Tessandier $X$, Amzalag G.

La décision en implantologie. Paris : CdP, 1993:30-73.

2. Berketa J, James $\mathrm{H}$, Marino V.

Dental implant changes following incineration. Forensic Sci Int 2011;207(1-3):50-54.

3. Berketa JW, Hirsch RS, Higgins D, James $\mathrm{H}$. Radiographic recognition of dental implants as an aid to identifying the deceased. J Forensic Sci 2010;55(1):66-70.

4. Cavézian R.

Évolution historique du concept implantaire : passé, présent et futur des moyens prédictifs d'imagerie. In : Actes Société française d'histoire de l'art dentaire. XVIII congrès. Castéra-Verduzan, 2007; 12:35-40.

5. Daas M, Dada K. Où en est l'implantologie en 2011 ? Inf Dent 2011;12(93):26-9.

6. Daas M, Dada K, Pompignoli M. Avis des industriels. Inf Dent 2011;12(93):147-50.

7. Davarpanah $\mathrm{M}$, Jakubowicz-Kohen B, Caraman M, Kebir-Quelin M. Les implants en Odontologie. Paris: CDP, 2004:39-45.

8. Davarpanah $\mathrm{M}$, Szmukler-Moncler S, Khoury PM, Jakubowicz-Kohen B, Martinez $\mathrm{H}$.
Manuel d'implantologie clinique.

Concepts, protocoles et innovations récentes. $2^{\mathrm{e}}$ édition.

Paris : CdP, 2008:1-12.

9. Donachie MJ Jr. Titanium: a technical guide. $2^{\text {nd }}$ ed. Materials Park Ohio: ASM International, 2000:1-2.

10. Grully H, Torres JH. Choisir un implant dentaire... mais à quel prix ? Comparaison des tarifs 2010 en France.

La Lettre de Stomatologie. 2011;49:35-7.

11. Michelinakis $G$, Scharrock $A$, Barclay CW. Identification of dental implant through the use of Implant Recognition Software (IRS). Int Dent J 2006;56(4):203-8. 
12. Nuzzolese $E$, Lusito $S$, Solarino B, Di Vella G. Radiographic dental implants recognition for geographic evaluation in human identification. J Forensic Odontostomatol 2008;26(1):8-11.

13. Perrier M. Introduction à l'odontostomatologie médico-légale. Rev Mens Suisse Odontostomatol 1998;108(3):237-46.

14. Pretty IA, Sweet $D$. A look at forensic dentistry. Part1: The role of teeth in the determination of human identity. Br Dent J 2001;190(7):359-66. Review.
15. Renouard F, Rangert B. Facteurs de risque et traitement implantaires. Paris : Quintessence International,1999:12-7.

16. Sahiwal IG, Woody RD, Benson BW, Guillen GE. Macro design morphology of endosseous dental implants. J Prosthet Dent 2002;87(5):543-51.

17. Sahiwal IG, Woody RD, Benson BW, Guillen GE. Radiographic identification of nonthreaded endosseous dental implants. J Prosthet Dent 2002;87(5):552-62.

18. Sahiwal IG, Woody RD, Benson BW, Guillen GE.
Radiographic identification of threaded endosseous dental implants. J Prosthet Dent 2002;87(5):563-77.

19. Sewerin I. Identification of dental implants on radiographs. Quintessence Int. 1992;23(9):611-8.

20. Van Noort R. Titanium: The implant material of today. J Master Sci 1987;22:3801-11.

21. Wemeau FG. Identification des implants dentaires en odontologie médico-légale.

Mémoire de DU d'Identification en Odontologie Médico-Légale. Nancy, 2010.

\section{SUMMARY}

\section{Identification of dental implants in forensic dentistry}

François-Georges WEMEAU Hugues THOMAS

Aline SUYBENG

Aimé CONIGLIARO

\section{Keywords \\ - implantology \\ - dental forensic identification \\ - forensic medecine \\ - recognition software}

Oral implantology is a discipline which has an important expansion since the $90^{\text {th }}$. No marking of theses implants is still present for the moment, although it exists for others like orthopedic ones, and we have little documentation on this subject.

Our study was based on the elaboration of the discovery of a dental implant, alone or in a body, through retroalveolar and panoramic dental X-rays, associated or not with an ablation of the maxillaries, and sometimes "implant in the hand".

Thanks to their presence, we can have lots of informations: health of the carrier, social status, age of the implant... The ways of analysis of theses dental implants have been presented by radiographic analysis thanks to data bases of the sites "what implant is that" and the IRS (Implant Recognition System). And we have elaborated a personnel study with identification register and logician which enable us to select 1 to 3 parameters in order to find a potential candidate.

This study about identification of dental implants shows the difficulty of this analysis and seems to confirm the importance of a competence in implantology and in legal odontology. No error or approximation must be made in this research. The explosion of the numbers and type of implants can be a problem, especially the case of the copy of the dental implants. At the end, it is highly necessary to complete and verify theses data bases, which is a complex and hard work. 


\section{Questions de connaissance}

>1. - Quels sont les renseignements que nous apportent

la présence d'implant(s) ?

- absence de contre-indication médicale à la pose implantaire (à un instant donné) ;

- possibilité financières supposée du fait du cout des implants (« population sans difficulté financière »);

- conception de l'implant et datation de celui-ci par rapport à l'évolution des techniques, ainsi qu'orientation géographique.
> 2. - Quels sont les moyens de reconnaissance d'implants dentaires?

- analyse morphologique et radiologique de l'implant ;

- présence de bases de données et de logiciels existants : " IRS » et " what implant is that $" . .$. ;

- possibilité d'aide par des opérateurs formés en médecine légale et en implantologie.

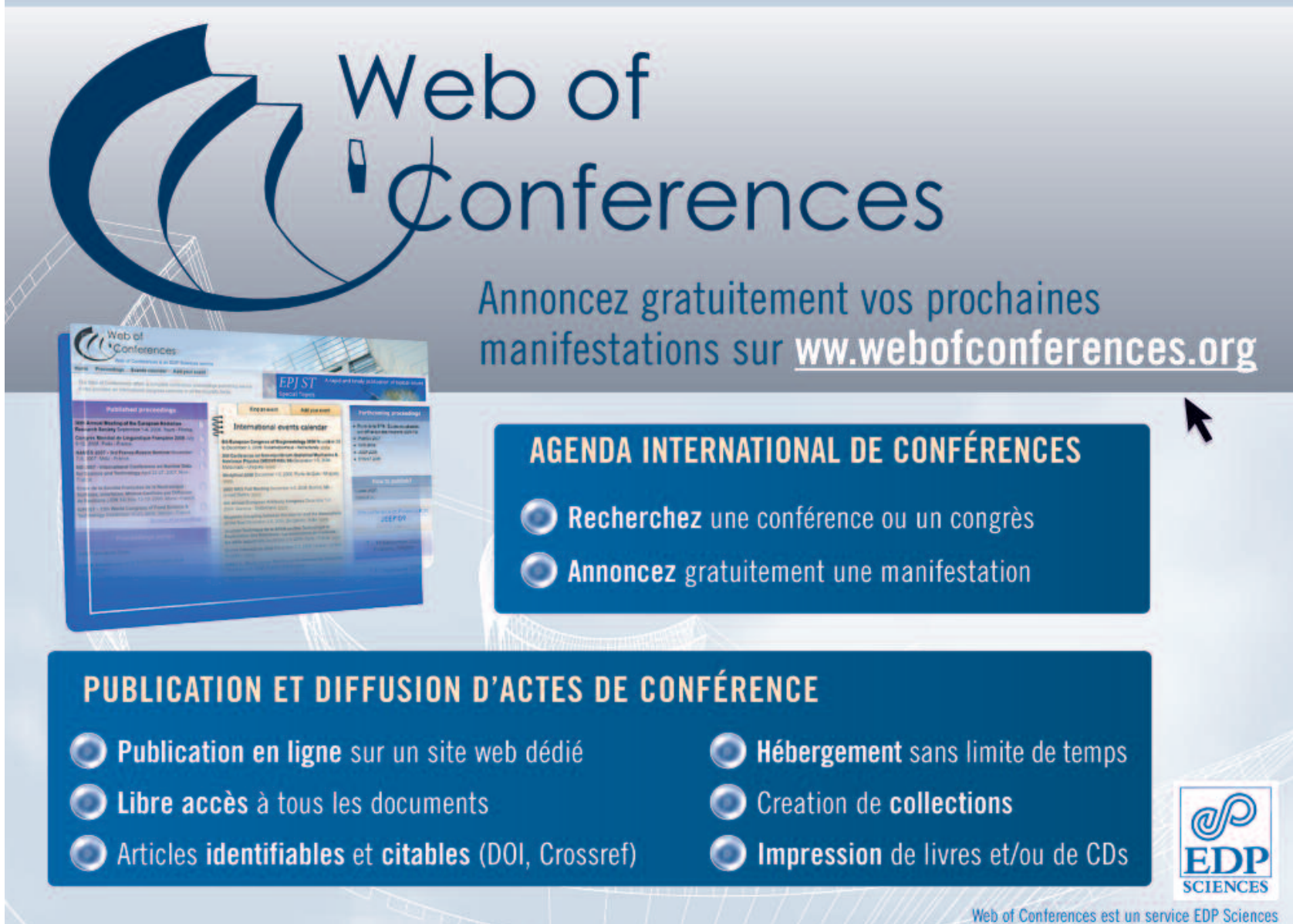

